\title{
Protective effects of Asiatic acid against pelvic inflammatory disease in rats
}

\author{
DEJIA KONG ${ }^{1}$, PING FU $^{2}$, QIN ZHANG ${ }^{2}$, XIAN MA $^{2}$ and PING JIANG ${ }^{2}$ \\ ${ }^{1}$ Department of Chinese Gynecology, Hangzhou Women's Hospital, Hangzhou, Zhejiang 310008; \\ ${ }^{2}$ Department of Chinese Gynecology, The Affiliated Guang-Xing Hospital of Zhejiang \\ Traditional Chinese Medicine University, Hangzhou, Zhejiang 310007, P.R. China
}

Received April 8, 2017; Accepted January 2, 2018

DOI: $10.3892 /$ etm.2019.7498

\begin{abstract}
Asiatic acid (AA) is one of the major components of the Chinese herb Centella asiatica and exerts a variety of pharmacological activities. However, the pharmacological effects of AA on pelvic inflammatory disease (PID) remain unknown. The purpose of the present study was to investigate the therapeutic efficacy and potential mechanisms of AA on PID in rats. A total of 75 female Sprague Dawley rats were randomly divided into the following five groups: A control group; a PID group; a $\mathrm{PID}+\mathrm{AA} 5 \mathrm{mg} / \mathrm{kg}$ group; a PID + AA $35 \mathrm{mg} / \mathrm{kg}$ group; and a $\mathrm{PID}+\mathrm{AA} 75 \mathrm{mg} / \mathrm{kg}$ group. Changes in cytokine and chemokine levels, myeloperoxidase (MPO) activity, nucleotide-binding domain-like receptor protein 3 (NLRP3) inflammasome and nuclear factor $-\kappa \mathrm{B}(\mathrm{NF}-\kappa \mathrm{B})$ activation, oxidative stress and cleaved caspase- 3 were measured. AA treatment significantly decreased the excessive production of cytokines and chemokines and suppressed MPO activity and the activation of NLRP3 inflammasome, $\mathrm{NF}-\kappa \mathrm{B}$ and caspase-3, as well as oxidative stress. These results suggest that AA exhibits potent anti-inflammatory and antioxidant effects in rats with pathogen-induced PID and that the mechanism of these anti-inflammatory effects may be associated with the suppression of NLRP3 inflammasome activation and the NF- $\kappa \mathrm{B}$ pathway.
\end{abstract}

\section{Introduction}

Pelvic inflammatory disease (PID) is a gynecological disease that is common among young and sexually active females with upper genital tract infections, including endometritis, salpingitis, tubal ovarian abscesses or pelvic peritonitis (1). Clinical

Correspondence to: Professor Ping Fu, Department of Chinese Gynecology, The Affiliated Guang-Xing Hospital of Zhejiang Traditional Chinese Medicine University, 453 Tiyuchang Road, Xihu, Hangzhou, Zhejiang 310007, P.R. China

E-mail: drfuping@163.com

Key words: asiatic acid, inflammatory response, pelvic inflammatory disease, nucleotide-binding domain-like receptor protein 3 inflammasome, nuclear factor- $\mathrm{kB}$ features of PID include adnexal pain, abnormal vaginal discharge, fever, menstrual irregularities and dyspareunia (2). Long-term and repeated infections lead to chronic pelvic pain, tubal infertility and ectopic pregnancy, which have a serious impact on health and quality of life $(3,4)$.

The pathophysiological mechanism of PID includes the inflammatory response $(5,6)$. The inflammatory response causes the release and maturation of interleukin (IL)-1 $\beta(5,6)$, which is mediated by nucleotide-binding domain-like receptor protein 3 (NLRP3) inflammasome $(7,8)$. NLRP3 inflammasome is composed of sensor NLRP3, adaptor protein apoptosis-associated speck-like protein and procaspase-1 $(7,8)$. The nuclear factor $-\kappa \mathrm{B}(\mathrm{NF}-\kappa \mathrm{B})$ pathway comprises important transcription factors that regulate inflammatory cytokines $(9,10)$ and chemokines $(11,12)$, including IL-1 $\beta$, IL-6, tumor necrosis factor- $\alpha$ (TNF- $\alpha)$ and monocyte chemotactic protein 1 (MCP-1), which are associated with the inflammatory responses of PID $(13,14)$. The clinical use of antibiotics is the preferred choice for PID treatment according to the Centers for Disease Control and Prevention guidelines in the United States (15); however, the majority of patients often experience subsequent bacterial drug-resistance (13). Therefore, the development of effective natural drugs for the prevention of PID is of great importance.

Asiatic acid (AA) is a natural triterpenoid extracted from Centella asiatica $(16,17)$. It has many beneficial properties, including anti-inflammatory (16) and antioxidant (18) effects. Recently, AA has been demonstrated to control inflammation and exert protective effects via inhibiting NLRP3 inflammasome activation (16) and the NF- $\mathrm{B}$ (19) pathway. However, the effects of AA on PID remain unknown. Therefore, the aim of present study was to investigate the protective effects and underlying mechanisms of AA in a rat model of PID.

\section{Materials and methods}

Chemicals and reagents. AA was purchased from Sigma-Aldrich (Merck KGaA; Darmstadt, Germany). Pentobarbital was purchased from Chengdu XiYa Chemical Technology Co.,Ltd.(Chengdu, China). Progesterone injections were obtained from Zhejiang Xianju Pharmaceutical Co., Ltd. (Taizhou, China). Absorbable gelatin sponges were purchased from Jinling Pharmaceutical Co., Ltd. (Nanjing, China). The 
pathogenic Escherichia coli strain and Ureaplasma urealyticum strain (t-strain mycoplasma) were obtained from Nanjing Bianzhen Biology Science and Technology Co., Ltd. (Nanjing, China).

Animal selection and group allocation. A total of 75 female specific pathogen-free Sprague Dawley rats (aged 9 weeks; weighing 220-240 g; Shanghai SLAC Laboratory Animal Co., Ltd., Shanghai, China), which were maintained under the controlled conditions at $23^{\circ} \mathrm{C}$ with a 12 -h light/dark cycle (60\% humidity) and access to food and water ad libitum, were assigned randomly to the following five equal groups: A control group, consisting of rats that received vehicle only (propylene glycol, $1.0 \mathrm{mg} / \mathrm{kg}$ ) via intragastric gavage once daily; a PID group consisting of rats that underwent the model of PID and received an equal volume of vehicle (propylene glycol, $1.0 \mathrm{mg} / \mathrm{kg}$ ) via intragastric gavage once daily; a PID + AA 5 group consisting of rats that underwent the model of PID and received $5 \mathrm{mg} / \mathrm{kg}$ AA in vehicle $(1.5 \mathrm{ml})$ via intragastric gavage once daily; a PID + AA 35 group consisting of rats that underwent the model of PID and received $35 \mathrm{mg} / \mathrm{kg} \mathrm{AA}$ in vehicle via intragastric gavage once daily; and a PID + AA 75 group consisting of rats that underwent the model of PID and received $75 \mathrm{mg} / \mathrm{kg} \mathrm{AA}$ in vehicle via intragastric gavage once daily. All procedures were approved by the Animal Ethics Committee of Zhejiang Chinese Medical University (Hangzhou, China).

Establishment of the PID model. A PID model in rats was established based on previously published protocols $(13,14)$. Rats in the study were immediately administered intramuscular injections of buprenorphine $(0.01 \mathrm{mg} / \mathrm{kg}$; bid; Tianjin Medicine Research Institute Pharmaceutical Co., Ltd., Tianjin, China) to relieve pain following infection (17). All rats were acclimated for 7 days and subcutaneously injected with $45 \mathrm{mg} / \mathrm{kg}$ progesterone (Zhejiang Xianju Pharmaceutical Co., Ltd., Taizhou, China) prior to infection. An absorbable gelatin sponge was saturated with microbe-mixing solution (Nanjing Bianzhen Biology Science and Technology Co., Ltd., Nanjing, China) with U. urealyticum $\left(1 \times 10^{8} \mathrm{cfu} / \mathrm{ml}\right)$ and pathogenic Escherichia coli $\left(1 \times 10^{8} \mathrm{cfu} / \mathrm{ml}\right)$. The upper genital tract of each rat in the PID group was then implanted with a microbe-containing gelatin sponge and rats were inverted for $3 \mathrm{~min}$. The cervixes of rats in the control group were implanted with microbe-free gelatin sponges. A total of four infections were performed every 2 days. Following the first infection, the experimental groups were administrated with AA via intragastric gavage and the PID and control groups were gavaged with an equal volume of vehicle. At 8 days following the first infection, rats were intravenously anesthetized with pentobarbital at a dose of $30 \mathrm{mg} / \mathrm{kg}$. Following anesthesia, the right fallopian tube and uterus were harvested and stored at $-80^{\circ} \mathrm{C}$. When rats exhibited severe symptoms e.g. convulsion for a period of $>10 \mathrm{~min}$, they were euthanized with via intravenous administration of pentobarbital at a dose of $140 \mathrm{mg} / \mathrm{kg}$ as previously described (20).

Western blot analysis. The right fallopian tube and right uterine horn ( $\mathrm{n}=5$ from each group) were used for western blotting. Samples were homogenized and extracted using radioimmunoprecipitation assay buffer (Beyotime Institute of Biotechnology, Nanjing, China), and then centrifuged at $5,000 \mathrm{x} \mathrm{g}$ at $4^{\circ} \mathrm{C}$ for $30 \mathrm{~min}$. The protein concentration in the supernatant was measured using a bicinchoninic acid assay kit. Soluble lysates (30 $\mu \mathrm{g} / \mathrm{lane})$ were resolved using $10 \%$ SDS-PAGE and transferred onto polyvinylidene difluoride membranes (EMD Millipore, Billerica, MA,USA). Membranes were blocked with $5 \%$ non-fat dry milk at $25^{\circ} \mathrm{C}$ for $2 \mathrm{~h}$ and incubation was performed with the following specific primary antibodies: Anti-NLRP3 (1:1,000; sc-34410), anti-caspase-1 (1:1,000; sc-1597 all Santa Cruz Biotechnology,Inc.,Dallas, TX, USA), anti-p-NF-кB p65 (1:500; ab10859369), anti-p-inhibitor

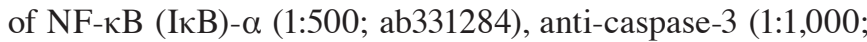
ab9664; all Abcam, Cambridge, UK) and anti- $\beta$-actin (1:1,000; sc-1616; Santa Cruz Biotechnology, Inc.) at $4^{\circ} \mathrm{C}$ overnight. Membranes were then rinsed with Tris-buffered saline with Tween and further incubated with the secondary antibody (goat anti-mouse IgG; 1:500; bs12478; Bioworld, Biogottechnology, Co., Ltd., Nanjing, China) for $1 \mathrm{~h}$ at room temperature. Detection of specific proteins was performed with an enhanced chemiluminescence kit (Thermo Fisher Scientific, Inc., Waltham, MA, USA). The levels of protein were analyzed using ImageJ (v2.1.4.7, National Institutes of Health, Bethesda, MD, USA) software.

Biochemical analysis. Samples of the right uterus and fallopian tube were collected and homogenized, and then centrifuged at $5,000 \mathrm{x}$ g at $4^{\circ} \mathrm{C}$ for $30 \mathrm{~min}$. The concentration of IL-1 $\beta$ (ERC007.96; Neobioscience, Beijing, China), IL-6 (ERC003.96; Neobioscience), TNF- $\alpha$ (ERC102a.96; Neobioscience), MCP-1 (ERC113.48; Neobioscience), chemokine C-X-C motif ligand 1 (CXCL-1; ab219044, Abcam) and chemokine C-C motif ligand 5 (RANTES; ERC105.96; Neobioscience,) supernatants was determined using ELISA kits according to the manufacturer's protocol. Malondialdehyde (MDA; S0131) production and superoxide dismutase (SOD; S0060) activity was measured by commercial kit (Beyotime Institute of Biotechnology) following the manufacturer's protocol. Myeloperoxidase (MPO) activity was assessed using an MPO assay kit (A044; Nanjing Jiancheng Bioengineering Institute, Nanjing, China).

Statistical analysis. Data are expressed as the mean + standard error of the mean and were analyzed through SPSS software (version 16.0, SPSS Inc., Chicago, IL, USA). Statistical analysis was performed using a one-way analysis of variance followed by Dunnett's post hoc test. $\mathrm{P}<0.05$ was determined to indicate a statistically significant difference.

\section{Results}

$A A$ reduces the level of inflammatory cytokines and chemokines. Levels of proinflammatory cytokines IL-1 $\beta$ (Fig. 1A), IL-6 (Fig. 1B) and TNF- $\alpha$ (Fig. 1C), as well as chemokines MCP-1 (Fig. 1D), RANTES (Fig. 1E) and CXCL-1 (Fig. 1F) were detected. The results indicate that IL-1 $\beta$, IL- 6 , TNF- $\alpha$, CXCL-1, MCP-1 and RANTES expression was significantly increased following infection in the PID group compared with the control group, whereas AA significantly decreased the expression of inflammatory cytokines and chemokines in 
A

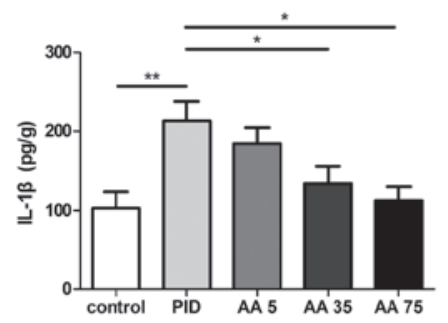

D

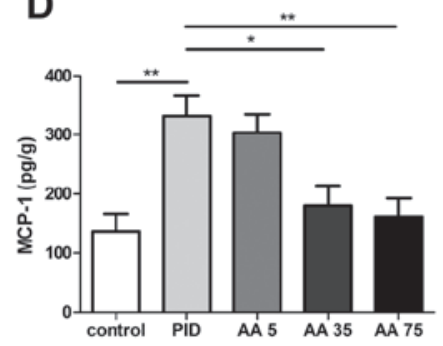

B

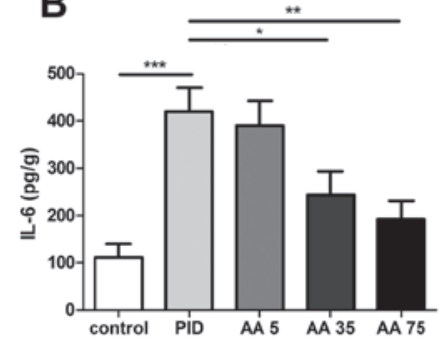

E

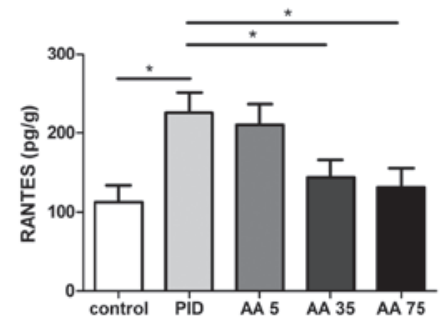

C

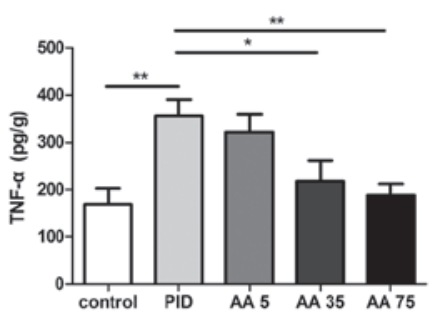

$\mathbf{F}$

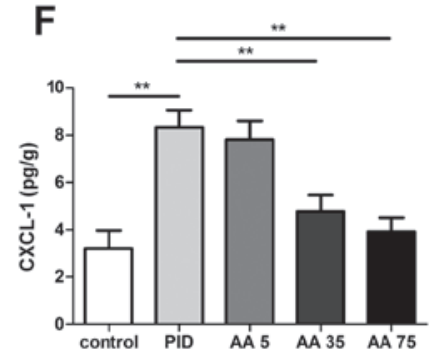

Figure 1. AA administration reduces the level of inflammatory cytokines and chemokines following the establishment of a PID model in rats. Effects of AA on the levels of (A) IL-1 $\beta$, (B) IL-6, (C) TNF- $\alpha$, (D) MCP-1, (E) RANTES and (F) CXCL-1. ${ }^{*} \mathrm{P}<0.05,{ }^{* *} \mathrm{P}<0.01,{ }^{* * *} \mathrm{P}<0.001 . \mathrm{n}=5$. AA, Asiatic acid; PID, pelvic inflammatory disease; IL, interleukin; TNF- $\alpha$, tumor necrosis factor- $\alpha$; MCP-1, monocyte chemotactic protein 1; RANTES, chemokine C-C motif ligand 5; CXCL-1, chemokine (C-X-C motif) ligand 1; AA 5, PID + 5 mg/kg AA group; AA 35, PID + 35 mg/kg AA group; AA 75, PID + 75 mg/kg AA group.

the PID + AA 35 and PID + AA 75 groups compared with the PID group (Fig. 1). No significant differences were observed between the PID and PID + AA 5 groups, PID + AA 35 and PID + AA 75 groups, PID + AA 35 and control groups, and PID + AA 75 and control groups.

AA inhibits neutrophil infiltration. MPO activity was assessed, as it is an oxidative enzyme in neutrophils and acts as a specific marker of neutrophil infiltration $(16,17,21)$ (Fig. 2). The results demonstrated that MPO activity was significantly increased in the PID group compared with the control group, whereas AA significantly inhibited MPO activity in the PID + AA 35 and PID + AA 75 groups compared with the PID group. The differences observed between the PID and PID + AA 5 groups, PID + AA 35 and PID + AA 75 groups, PID + AA 35 and control groups, and PID + AA 75 and control groups were not significant.

AA suppresses NLRP3 inflammasome activation and the $N F-\kappa B$ pathway. To determine the potential mechanisms of AA, the expression of NLRP3, caspase-1 p-NF- $\mathrm{B}$ p 65 , p-IкB- $\alpha$, and cleaved caspase- 3 were measured. PID induced a significantly higher expression of NLRP3, active caspase-1, p-NF- $\kappa$ B p 65, p-IкB- $\alpha$ and cleaved caspase-3 protein in the PID group compared with the control group (Fig. 3). By contrast, these changes were significantly reversed in the PID + AA 35 and PID + AA 75 groups compared with the PID group. However, the levels of active caspase-1, p-NF- $\kappa$ B p65, p-IкB- $\alpha$ and cleaved caspase-3 (only in the PID + AA 35 group) were significantly increased in the PID + AA 35 and PID + AA 75 groups relative to control animals. Furthermore, the differences observed between PID + AA 35 and control groups, and PID + AA 75 and control group on the level of NLRP3 were not significant. No significant differences were observed between the PID and PID + AA 5 groups or the PID + AA 35 and PID + AA 75 groups.

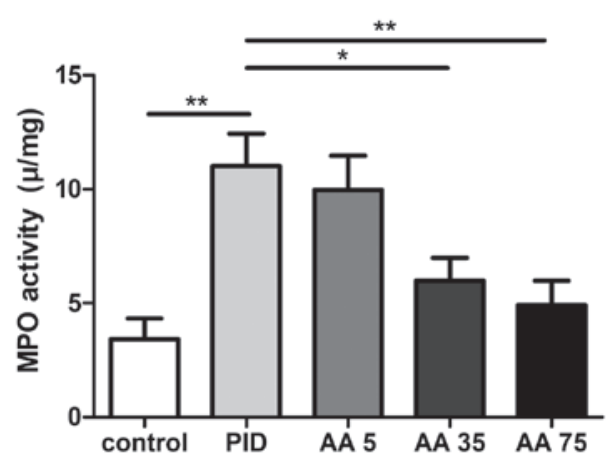

Figure 2. AA administration inhibits neutrophil infiltration. Effect of AA on MPO activity. ${ }^{*} \mathrm{P}<0.05,{ }^{* *} \mathrm{P}<0.01$. $\mathrm{n}=5$. AA, Asiatic acid; MPO, myeloperoxidase; PID, pelvic inflammatory disease; AA 5, PID + $5 \mathrm{mg} / \mathrm{kg}$ AA group; AA 35, PID $+35 \mathrm{mg} / \mathrm{kg}$ AA group; AA 75, PID $+75 \mathrm{mg} / \mathrm{kg}$ AA group.

AA attenuates oxidative stress. The effects of AA on SOD activity and MDA production were assessed (Fig. 4). There was a significant decrease in SOD activity in the PID group compared with the control group, whereas the MDA level was significantly increased. However, treatment with AA in the PID + AA 35 and PID + AA 75 groups significantly increased the SOD activity and decreased the production of MDA compared with the PID group. However, the levels of MDA were significantly increased in the PID + AA 35 group relative to control animals. No significant differences were observed between the PID and PID + AA 5 groups, the PID + AA 35 and PID + AA 75 groups, and PID + AA 75 and control groups.

\section{Discussion}

AA is derived from a Chinese herbal plant, which has a long medical history in China $(16,17)$. Due to its low side-effect profile and range of biological properties, it has been used to treat a wide range of diseases (22-24). However, the effects of 


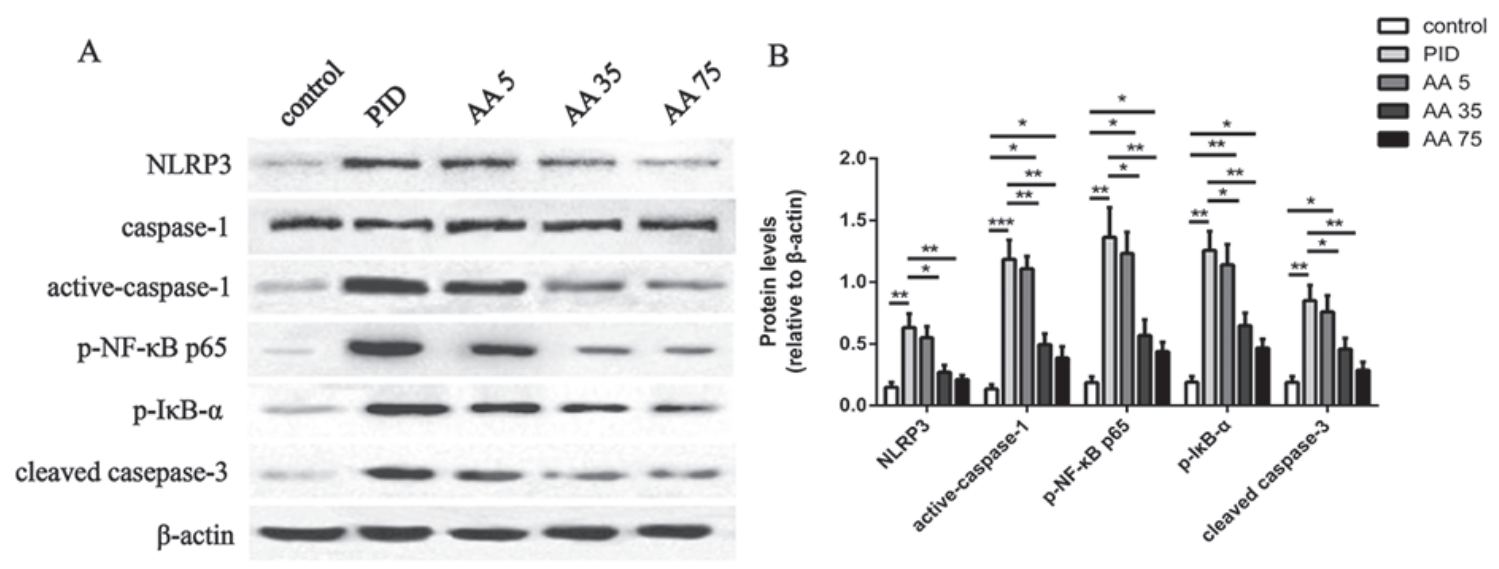

Figure 3. AA administration suppresses activation of NLRP3 inflammasome, the NF-kB pathway and caspase-3. (A) Levels of NLRP3, caspase 1, active-caspase-1, p-NF-кB p65, p-IкB- $\alpha$, cleaved caspase- 3 and $\beta$-actin indicated by western blotting. (B) Quantification of western blotting. " $\mathrm{P}<0.05$, ${ }^{* *} \mathrm{P}<0.01$,

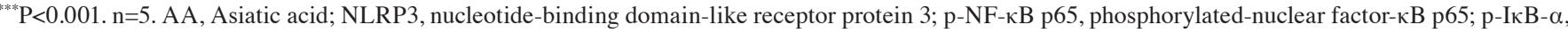
phosphorylated- inhibitor of NF-kB; PID, pelvic inflammatory disease; AA 5, PID + $5 \mathrm{mg} / \mathrm{kg}$ AA group; AA 35, PID + $35 \mathrm{mg} / \mathrm{kg} \mathrm{AA} \mathrm{group;} \mathrm{AA} \mathrm{75,}$ $\mathrm{PID}+75 \mathrm{mg} / \mathrm{kg}$ AA group.

A

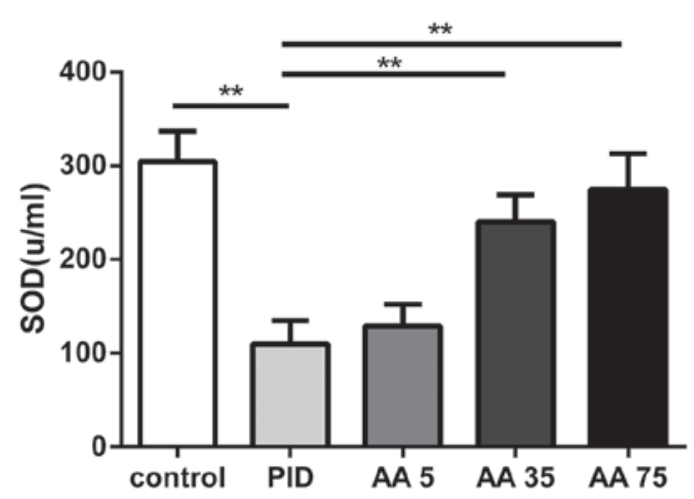

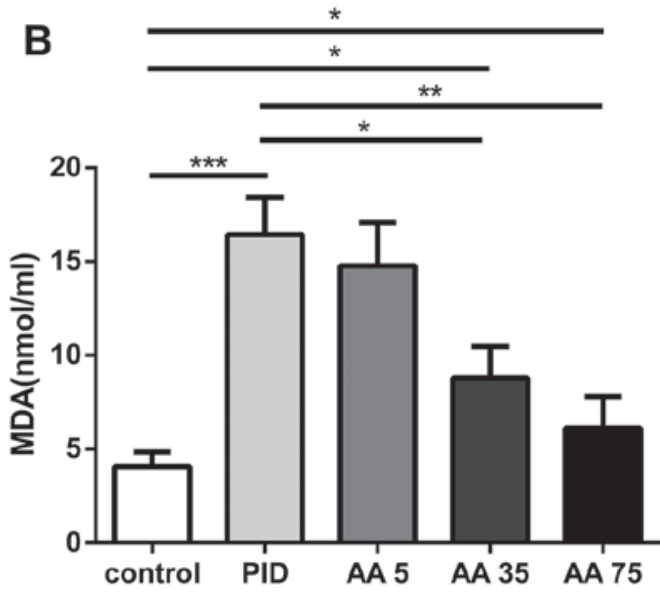

Figure 4. AA administration attenuates oxidative stress. Effects of AA on (A) MDA production and (B) SOD activity. ${ }^{*} \mathrm{P}<0.05,{ }^{* *} \mathrm{P}<0.01,{ }^{* * * *} \mathrm{P}<0.001$. $\mathrm{n}=5$. AA, Asiatic acid; MDA, malondialdehyde; SOD, superoxide dismutase; PID, pelvic inflammatory disease; AA 5, PID + $5 \mathrm{mg} / \mathrm{kg}$ AA group; AA 35 , PID + 35 mg $/ \mathrm{kg}$ AA group; AA 75, PID + $75 \mathrm{mg} / \mathrm{kg}$ AA group.

AA on PID remain unknown. The results of the present study demonstrated that AA decreases the levels of inflammatory cytokines and chemokines, inhibits neutrophil infiltration, suppresses the activation of NLRP3 inflammasome, caspase-3 and the NF- $\mathrm{kB}$ pathway, and attenuates oxidative stress.

Inflammation is a primordial defense against infection (25); however, an excessive inflammatory response may induce tissue damage and cause physiological dysfunction $(16,17)$. IL- $1 \beta$ is a subtype of IL-1 and a pivotal inflammatory cytokine that increases levels of proinflammatory cytokines, including IL-6 and TNF- $\alpha$, amplifies the inflammatory response and induces apoptosis (26). IL-6 is another principal proinflammatory cytokine that serves an important role, regulating the release of chemotactic mediators and cell adhesion molecules (27). Furthermore, IL-6 directly affects tubal transport (28). TNF- $\alpha$ also contributes to cell death and tissue injury $(28,29)$. In addition, chemokines CXCL-1, MCP-1 and RANTES serve a role in the recruitment and activation of inflammatory cells, and activated neutrophils promote the inflammatory response (13).
In the present study, levels of proinflammatory cytokines, chemokines and neutrophils were significantly increased following pathogen infection and AA treatment markedly reversed these changes.

NLRP3 inflammasome serves an important role in inflammation $(7,8,15)$. Aberrant NLRP3 inflammasome activation is deleterious and conducive to the development of a number of inflammatory diseases (30). The aim of the present study was therefore to evaluate its relevance during PID. To the best of our knowledge, the current study is the first to demonstrate that PID induces NLRP3 inflammasome activation, while AA administration suppresses it.

Oxidative stress, including reactive oxygen species (ROS), contributes to cell and tissue damage; furthermore, ROS regulate NLRP3 inflammasome activation (31). SOD is an important antioxidant enzyme that clears oxygen radicals and inhibits tissue damage (32). MDA is the final product of lipid peroxidation, which represents the oxidative stress intensity and oxygen free radical levels (17). The results of the present study suggest that 
PID reduces SOD and increase MDA, whereas AA treatment markedly inhibits these changes and attenuates oxidative stress.

In conclusion, the results of the present study suggest that AA treatment has potent anti-inflammatory and antioxidant effects in rats with pathogen-induced PID and the mechanism of this action may be associated with suppression of NLRP3 inflammasome activation and the NF- $\mathrm{NB}$ pathway. The present study provides a basis for further research into the potential use of AA as a clinical treatment for patients with PID.

\section{Acknowledgements}

Not applicable.

\section{Funding}

This study was supported by the National Famous Old Chinese Medicine Experts Inheritance Studio Project of State Administration of Traditional Chinese Medicine (grant no. 2014[20]). Fuping Famous Old Chinese Medicine Experts Inheritance Studio Research Project of Zhejiang Province (grant no. GZS2012023).

\section{Availability of data and materials}

The datasets used and/or analyzed during the current study are available from the corresponding author on reasonable request.

\section{Authors' contributions}

$\mathrm{DK}$ and PF were responsible for the experimental design and data analysis. DK drafted the manuscript. QZ and XM were responsible for the experiments. PJ analyzed amd interpreted the data and revised the manuscript critically for important intellectual content. All authors checked and approved the final manuscript.

\section{Ethics approval and consent to participate}

All animal procedures and care were approved by the guidelines of Animal Ethics Committee of Zhejiang Chinese Medical University and were in compliance with the relevant laws and institutional guidelines.

\section{Patient consent for publication}

Not applicable.

\section{Competing interests}

The authors declare that they have no competing interests.

\section{References}

1. Gradison M: Pelvic inflammatory disease. Am Fam Physician 85 791-796, 2012.

2. Newton D, Bayly C, Fairley CK, Chen M, Keogh L, Temple-Smith M, Williams H, McNamee K, Fisher J, Henning D, et al: Women's experiences of pelvic inflammatory disease: Implications for health-care professionals. J Health Psychol 19: 618-628, 2014.
3. Ross JD: Pelvic inflammatory disease. BMJ Clin Evid 2013: 1606, 2013

4. Bu X, Liu Y, Lu Q and Jin Z: Effects of 'Danzhi Decoction' on chronic pelvic pain, hemodynamics, and proinflammatory factors in the murine model of sequelae of pelvic inflammatory disease. Evid Based Complement Alternat Med 2015: 547251, 2015.

5. Dhasmana D, Hathorn E, McGrath R, Tariq A and Ross JD: The effectiveness of nonsteroidal anti-inflammatory agents in the treatment of pelvic inflammatory disease: A systematic review. Syst Rev 3: 79, 2014

6. Tee YT, Wang PH, Yang SF, Tsai HT, Lee SK, Ko JL, Lin LY and Chen SC: Correlation of plasma osteopontin and neutrophil gelatinase-associated lipocalin levels with the severity and clinical outcome of pelvic inflammatory disease. Taiwan J Obstet Gynecol 53: 158-161, 2014.

7. Kayagaki N, Stowe IB, Lee BL, O'Rourke K, Anderson K, Warming S, Cuellar T, Haley B, Roose-Girma M, Phung QT, et al: Caspase-11 cleaves gasdermin D for non-canonical inflammasome signalling. Nature 526: 666-671, 2015.

8. Lamkanfi $\mathrm{M}$ and Dixit VM: Mechanisms and functions of inflammasomes. Cell 157: 1013-1022, 2014.

9. Lin TH, Yao Z, Sato T, Keeney M, Li C, Pajarinen J, Yang F, Egashira K and Goodman SB: Suppression of wear-particle-induced pro-inflammatory cy tokine and chemokine production in macrophages via NF- $\mathrm{BB}$ decoy oligodeoxynucleotide: A preliminary report. Acta Biomater 10: 3747-3755, 2014.

10. Han L, Sun J, Lu CJ, Zhao RZ, Lu Y, Lin HJ and Wei JA: Formula PSORI-CM01 inhibits the inflammatory cytokine and chemokine release in keratinocytes via NF- $\kappa \mathrm{B}$ expression. Int Immunopharmacol 44: 226-233, 2017.

11. Karkeni E, Bonnet L, Astier J, Couturier C, Dalifard J, Tourniaire F and Landrier JF: All-trans-retinoic acid represses chemokine expression in adipocytes and adipose tissue by inhibiting NF- $\kappa$ B signaling. J Nutr Biochem 42: 101-107, 2017.

12. Burke SJ, Stadler K, Lu D, Gleason E, Han A, Donohoe DR, Rogers RC, Hermann GE, Karlstad MD and Collier JJ: IL-1 $\beta$ reciprocally regulates chemokine and insulin secretion in pancreatic $\beta$-cells via NF- $\kappa$ B. Am J Physiol Endocrinol Metab 309: E715-E726, 2015.

13. Zou W, Xiao Z, Wen X, Luo J, Chen S, Cheng Z, Xiang D, $\mathrm{Hu} \mathrm{J}$ and $\mathrm{He} \mathrm{J}$ : The anti-inflammatory effect of Andrographis paniculata (Burm. f.) Nees on pelvic inflammatory disease in rats through down-regulation of the NF- $\kappa \mathrm{B}$ pathway. BMC Complement Altern Med 16: 483, 2016.

14. Zou W, Wen X, Sheng X, Zheng YI, Xiao Z, Luo J, Chen S, Wang Y, Cheng Z, Xiang D and Nie Y: Gas chromatography-mass spectrometric method-based urine metabolomic profile of rats with pelvic inflammatory disease. Exp Ther Med 11: 1653-1660, 2016.

15. Workowski KA and Bolan GA; Centers for Disease Control and Prevention: Sexually transmitted diseases treatment guidelines, 2015. MMWR Recomm Rep 64: 64: 1-137, 2015.

16. Jiang W, Li M, He F, Bian Z, He Q, Wang X, Yao W and Zhu L: Neuroprotective effect of asiatic acid against spinal cord injury in rats. Life Sci 157: 45-51, 2016.

17. Jiang W, Li M,He F, Yao W, Bian Z, Wang X and Zhu L: Protective effects of asiatic acid against spinal cord injury-induced acute lung injury in rats. Inflammation 39: 1853-1861, 2016.

18. Pakdeechote P, Bunbupha S, Kukongviriyapan U, Prachaney P, Khrisanapant W and Kukongviriyapan V: Asiatic acid alleviates hemodynamic and metabolic alterations via restoring eNOS/iNOS expression, oxidative diet-induced metabolic syndrome rats. Nutrients 6: 355-370, 2014.

19. Lee JW, Park HA, Kwon OK, Jang YG, Kim JY, Choi BK, Lee HJ, Lee S, Paik JH, Oh SR, et al: Asiatic acid inhibits pulmonary inflammation induced by cigarette smoke. Int Immunopharmacol 39: 208-217, 2016.

20. Morton DB and Griffiths PH: Guidelines on the recognition of pain, distress and discomfort in experimental animals and an hypothesis for assessment. Vet Rec 116: 431-436, 1985.

21. Liu J, Yi L, Xiang Z, Zhong J, Zhang H and Sun T: Resveratrol attenuates spinal cord injury-induced inflammatory damage in rat lungs. Int J Clin Exp Pathol 8: 1237-1246, 2015.

22. Guo W, Liu W, Jin B, Geng J, Li J, Ding H, Wu X, Xu Q, Sun Y and Gao J: Asiatic acid ameliorates dextran sulfate sodium-induced murine experimental colitis via suppressing mitochondria-mediated NLRP3 inflammasome activation. Int Immunopharmacol 24: 232-238, 2015.

23. Li Z, Xiao X and Yang M: Asiatic acid inhibits lipopolysaccharide-induced acute lung injury in mice. Inflammation 39: 1642-1648, 2016. 
24. Hao C, Wu B, Hou Z, Xie Q, Liao T, Wang T and Ma D: Asiatic acid inhibits LPS-induced inflammatory response in human gingival fibroblasts. Int Immunopharmacol 50: 313-318, 2017.

25. Gilroy DW: Eicosanoids and the endogenous control of acute inflammatory resolution. Int J Biochem Cell Biol 42: 524-528, 2010.

26. Jiang W, Huang Y, He F, Liu J, Li M, Sun T, Ren W, Hou J and Zhu L: Dopamine D1 receptor agonist A-68930 inhibits NLRP3 inflammasome activation, controls inflammation, and alleviates histopathology in a rat model of spinal cord injury. Spine (Phila Pa 1976) 41: E330-E334, 2016.

27. Kamimura D, Ishihara $K$ and Hirano T: IL-6 signal transduction and its physiological roles: The signal orchestration model. Rev Physiol Biochem Pharmacol 149: 1-38, 2003.

28. Lee SA, Tsai HT, Ou HC, Han CP, Tee YT, Chen YC, Wu MT, Chou MC, Wang PH and Yang SF: Plasma interleukin-1beta, $-6,-8$ and tumor necrosis factor-alpha as highly informative markers of pelvic inflammatory disease. Clin Chem Lab Med 46: 997-1003, 2008.
29. Dash SK, Chattopadhyay S, Dash SS, Tripathy S, Das B, Mahapatra SK, Bag BG, Karmakar P and Roy S: Self assembled nano fibers of betulinic acid: A selective inducer for ROS/TNF-alpha pathway mediated leukemic cell death. Bioorg Chem 63: 85-100, 2015.

30. Baldwin AG, Brough D and Freeman S: Inhibiting the inflammasome: A chemical perspective. J Med Chem 59: 1691-1710, 2016.

31. Ding W, Guo H, Xu C, Wang B, Zhang M and Ding F: Mitochondrial reactive oxygen species-mediated NLRP3 inflammasome activation contributes to aldosterone-induced renal tubular cells injury. Oncotarget 7: 17479-17491, 2016.

32. Jing W, Chunhua M and Shumin W: Effects of acteoside on lipopolysaccharide-induced inflammation in acute lung injury via regulation of NF- $\kappa \mathrm{B}$ pathway in vivo and in vitro. Toxicol Appl Pharmacol 285: 128-135, 2015. 